\title{
Determination of elastic modulus in nickel alloy from ultrasonic measurements
}

\author{
NIKHAT PARVEEN and G V S MURTHY* \\ Materials Science and Technology Division, National Metallurgical Laboratory, Jamshedpur 831 007, India
}

MS received 2 December 2008; revised 28 January 2011

\begin{abstract}
Elastic constants relate technological, structural and safety aspects to various materials phenomena and to their fundamental interatomic forces. Hence, they are of fundamental importance in almost all engineering applications. Thus its determination is of utmost importance. The aim of the present investigation is to study the behaviour of elastic constants and the variation on heat treatment in a nickel base super alloy Nimonic 263 by ultrasonic velocity measurements. From the present study it is evident that the elastic moduli of the material are very sensitive to any minor compositional changes, resulting due to the formation of intermetallic phases on heat treatment and can be effectively monitored by ultrasonic.
\end{abstract}

Keywords. Elastic constants; ultrasonic; nickel alloy; precipitation.

\section{Introduction}

An elastic modulus or modulus of elasticity is the mathematical description of a material or component's tendency to be deformed elastically when a force is applied to it. It is defined as the slope of the stress-strain curve in the elastic deformation region. Stress in turn is defined as the force causing the deformation divided by the area to which the force is applied and strain is the ratio of the change caused by stress to the original state of the object.

Elastic constants relate to various fundamental solid-state phenomena, including interatomic potentials, equations of state, and phonon spectra. They link thermodynamically with specific heat, thermal expansivity, the Debye temperature, and Grüneisen's constant. In engineering design they appear in calculations for load deflection, residual stress, thermoelastic stress, fracture toughness and elastic instabilities such as buckling and jamming. So, elastic constants are many things to everybody: they entice structural designers, materials scientists, and solid-state theorists; they connect to technological, structural economics and safety, to various materials phenomena and to their fundamental interatomic forces (Ledbetter 1983). In any material which is a multiphase alloy, the elastic modulus is determined by the modulus of the individual phases and by their relative volume fractions. Elastic modulus is more sensitive to the phase/crystal structure than any other factors (Long and Rack 1997; Ho et al 1999; Moorthy et al 2003; Majumdar et al 2008).

Thus it is imperative to determine the elastic properties of materials in its many ways. The elastic moduli can be determined by different methods. The different methods for the

*Author for correspondence (gvs@nmlindia.org) determination of the elastic constants can be classified as direct and indirect methods, as well as static and dynamic methods. In direct methods, these constants are determined from the longitudinal and transverse deformations as well as by the effects which depend directly on them, whereas in the indirect methods these are determined from the measurements of other elastic constants. In static methods, these constants are determined by the deformations produced by the time dependent or slowly changing forces in contrast to dynamic methods which often involve vibration studies.

Ultrasound is one example of both indirect and dynamic methods for the determination of elastic constants. It is based on the time of travel of ultrasonic impulses. A single impulse from the transmitter causes both a longitudinal and a transverse wave and the velocity of each is measured. It is reported that accuracy of such determinations varied between $3 \%$ and 5\% (Ledbetter 1983).

Using the following empirical formulae (McIntire 1991), different elastic moduli can be determined from the measurement of the ultrasonic longitudinal and shear-wave velocities and $\rho$, the density of the material:

$$
\begin{aligned}
& G=\rho V_{\mathrm{T}}^{2}, \\
& B=\rho\left(V_{\mathrm{L}}^{2}-\frac{4}{3} V_{\mathrm{T}}^{2}\right), \\
& E=\frac{3 \rho V_{\mathrm{T}}^{2}\left(V_{\mathrm{L}}^{2}-\frac{4}{3} V_{\mathrm{T}}^{2}\right)}{\left(V_{\mathrm{L}}^{2}-V_{\mathrm{T}}^{2}\right)},
\end{aligned}
$$

where $G, B$ and $E$ are the shear modulus, bulk modulus, and Young's modulus, respectively and $V_{\mathrm{L}}$ and $V_{\mathrm{T}}$ are the 
ultrasonic longitudinal and shear wave velocities, respectively. These equations form the basis for materials characterization by ultrasonic velocity measurements and are used for studying the effect of metallurgical processes like precipitation. Based on the above formulae, various elastic constants are calculated by substituting the ultrasonic parameters.

The aim of the present investigation is to study the behaviour of elastic constants and the variation on heat treatment in a nickel base super alloy Nimonic 263 by ultrasonic velocity measurements.

Nimonic 263 is a wrought Ni-based super alloy, which has found applications in gas turbines because of its attractive strength and good oxidation resistance. It was developed and introduced by Rolls Royce in 1960. It was designed primarily for use in the stationary components like combustion chamber, casing, exhaust ducting, bearing housing and many others. These components are fabricated from plate/sheet of this alloy. It is thus evident that the physical properties should be monitored as and when the components are being formed to attain the designed structural properties.

\section{Experimental}

Table 1 shows the elemental composition of the alloy used in the present study. A set of Nimonic 263 specimens of dimensions $20 \times 20 \times 10 \mathrm{~mm}$ was solutionized at $1423 \mathrm{~K}$ for $1 \mathrm{~h}$ followed by water quenching. Ageing was carried out on the solutionized samples for 1, 2, 4, 6 and $8 \mathrm{~h}$ at $923 \mathrm{~K}$ and 1, 2, $4,6,25,50$ and $75 \mathrm{~h}$ at $1073 \mathrm{~K}$ followed by water quenching. The samples are designated as ' $\mathrm{S}$ ' for solutionized only,
'A1' solutionized and aged at $923 \mathrm{~K}$ for $1 \mathrm{~h}$ so on up to 'A5' solutionized and aged at $923 \mathrm{~K}$ for $8 \mathrm{~h}$. Similarly 'B1' represents solutionized and aged at $1073 \mathrm{~K}$ for $1 \mathrm{~h}$, and so on up to 'B7' solutionized and aged at $1073 \mathrm{~K}$ for $75 \mathrm{~h}$.

The experimental set up used for the ultrasonic measurements consists of a $35 \mathrm{MHz}$ broadband pulser-receiver and a $500 \mathrm{MHz}$ digital oscilloscope. From these measurements, the RF signals were digitized and the gated back wall echoes from the oscilloscope were stored for further processing. Ultrasonic velocities were measured using $15 \mathrm{MHz}$ longitudinal wave and $5 \mathrm{MHz}$ shear wave transducers. A crosscorrelation technique has been used for precise ultrasonic velocity measurements (Kumar et al 2003). The accuracy obtained in the time-of-flight measurement is better than $\pm 1 \mathrm{~ns}$. This led to the maximum scatter of $\pm 2.5 \mathrm{~m} / \mathrm{s}$ and $\pm 1.5 \mathrm{~m} / \mathrm{s}$ for ultrasonic longitudinal and shear wave velocities, respectively. The density of the material was assumed to be constant in the present study.

\section{Results and discussion}

Based on the above formulae, various elastic modulus were calculated by substituting the ultrasonic parameters. The results are presented in table 2. The Young's modulus as evaluated here from the ultrasonic velocity measurements is in good agreement with the reported value (Betteridge and Heslop 1974). The modulus of the solution-annealed sample is the lowest and it increases with aging at $923 \mathrm{~K}$ and $1073 \mathrm{~K}$. This increase in the moduli upon thermal ageing

Table 1. Chemical composition of Nimonic 263.

\begin{tabular}{lcccccccccr}
\hline Element & $\mathrm{C}$ & $\mathrm{Co}$ & $\mathrm{Cr}$ & $\mathrm{Mo}$ & $\mathrm{Ti}$ & $\mathrm{Al}$ & $\mathrm{Cu}$ & $\mathrm{O}$ & $\mathrm{N}$ & $\mathrm{Ni}$ \\
\hline $\mathrm{Wt} \%$ & 0.06 & 19.6 & 20.1 & 6.0 & 2.0 & 0.4 & 0.001 & 0.0013 & 0.006 & $\mathrm{Bal}$ \\
\hline
\end{tabular}

Table 2. Ultrasonic velocities, and elastic properties of Nimonic 263 in different heat treatment conditions.

\begin{tabular}{|c|c|c|c|c|}
\hline Heat treatment & $\begin{array}{l}\text { Longitudinal wave } \\
\text { velocity }(\mathrm{m} / \mathrm{s})\end{array}$ & $\begin{array}{c}\text { Shear wave } \\
\text { velocity }(\mathrm{m} / \mathrm{s})\end{array}$ & $\begin{array}{c}\text { Shear } \\
\text { modulus (GPa) }\end{array}$ & $\begin{array}{c}\text { Young's } \\
\text { modulus (GPa) }\end{array}$ \\
\hline$S$ & $5960 \pm 2 \cdot 5$ & $3155 \pm 1 \cdot 5$ & $83 \cdot 2$ & $217 \cdot 3$ \\
\hline A1 & $5981 \pm 2 \cdot 5$ & $3170 \pm 1 \cdot 5$ & $84 \cdot 0$ & $219 \cdot 2$ \\
\hline $\mathrm{A} 2$ & $5980 \pm 2 \cdot 5$ & $3175 \pm 1 \cdot 5$ & $84 \cdot 3$ & $219 \cdot 7$ \\
\hline A 3 & $5992 \pm 2 \cdot 5$ & $3187 \pm 1 \cdot 5$ & $84 \cdot 7$ & $221 \cdot 2$ \\
\hline A4 & $5993 \pm 2 \cdot 5$ & $3185 \pm 1 \cdot 5$ & $84 \cdot 8$ & 221.4 \\
\hline A5 & $5995 \pm 2 \cdot 5$ & $3192 \pm 1 \cdot 5$ & $85 \cdot 2$ & $221 \cdot 8$ \\
\hline B1 & $5984 \pm 2 \cdot 5$ & $3181 \pm 1 \cdot 5$ & $84 \cdot 8$ & $220 \cdot 7$ \\
\hline B2 & $5982 \pm 2 \cdot 5$ & $3179 \pm 1 \cdot 5$ & $84 \cdot 4$ & $220 \cdot 0$ \\
\hline B3 & $5987 \pm 2 \cdot 5$ & $3183 \pm 1 \cdot 5$ & $84 \cdot 5$ & $220 \cdot 7$ \\
\hline B4 & $5988 \pm 2 \cdot 5$ & $3180 \pm 1 \cdot 5$ & $84 \cdot 5$ & $220 \cdot 4$ \\
\hline B5 & $5987 \pm 2 \cdot 5$ & $3183 \pm 1 \cdot 5$ & $84 \cdot 7$ & $220 \cdot 7$ \\
\hline B6 & $5986 \pm 2 \cdot 5$ & $3182 \pm 1 \cdot 5$ & $84 \cdot 7$ & $220 \cdot 6$ \\
\hline B7 & $5985 \pm 2 \cdot 5$ & $3181 \pm 1 \cdot 5$ & $84 \cdot 6$ & $220 \cdot 5$ \\
\hline
\end{tabular}


is attributed to the formation of the $\gamma^{\prime}$ precipitates leading to the depletion of the precipitate-forming elements from the solution. It was also noticed that the changes in elastic moduli (about $1.54 \%$ in YS and $1.72 \%$ in shear moduli) are minor. Thus it is evident that the minor changes in elastic moduli of the material are very sensitive to any minor compositional changes, resulting from the formation of intermetallic phases on heat treatment, and can be effectively monitored by ultrasonic.

The solution-annealed specimen exhibited the lowest modulus (83.2 MPa) and it increased with ageing at $923 \mathrm{~K}$ beyond $1 \mathrm{~h}$ of ageing and at $1073 \mathrm{~K}$ from $1 \mathrm{~h}$ onwards. Upon ageing at $923 \mathrm{~K}$, the modulus was found to increase continuously up to the maximum duration of ageing $(8 \mathrm{~h})$ used in the present investigation. Upon ageing at $1073 \mathrm{~K}$, modulus increased rapidly up to $6 \mathrm{~h}$, followed by a subtle increase up to $50 \mathrm{~h}$ followed by a small decrease at $75 \mathrm{~h}$ of ageing. The increase in the modulus at both the temperatures is attributed to the precipitation of coherent $\gamma^{\prime}$ intermetallic phase. The shorter incubation period and faster increase during the initial period of ageing (up to $6 \mathrm{~h}$ ) at $1073 \mathrm{~K}$ as compared to those at $923 \mathrm{~K}$ is attributed to the faster kinetics of precipitation at higher temperature $(1073 \mathrm{~K})$. The results obtained are in line with the time temperature transformation (TTT) diagram reported by Zhao and Henry (2002) for the precipitation of $\gamma^{\prime}$ intermetallic phase in this alloy. The variation in modulus in concert with ultrasonic velocity on ageing time and temperature is also confirmed with the electron microscopy studies. This is evident from the microstructure of the sample aged at $1073 \mathrm{~K}$ for $50 \mathrm{~h}$ which shows extensive precipitation of coherent $\gamma^{\prime}$ intermetallic phase (figure 1). Moreover, it is appropriate to note that even though there is extensive precipitation as seen in the microstructure, the change in the modulus is only minor (about $1.5 \%$ only). Thus

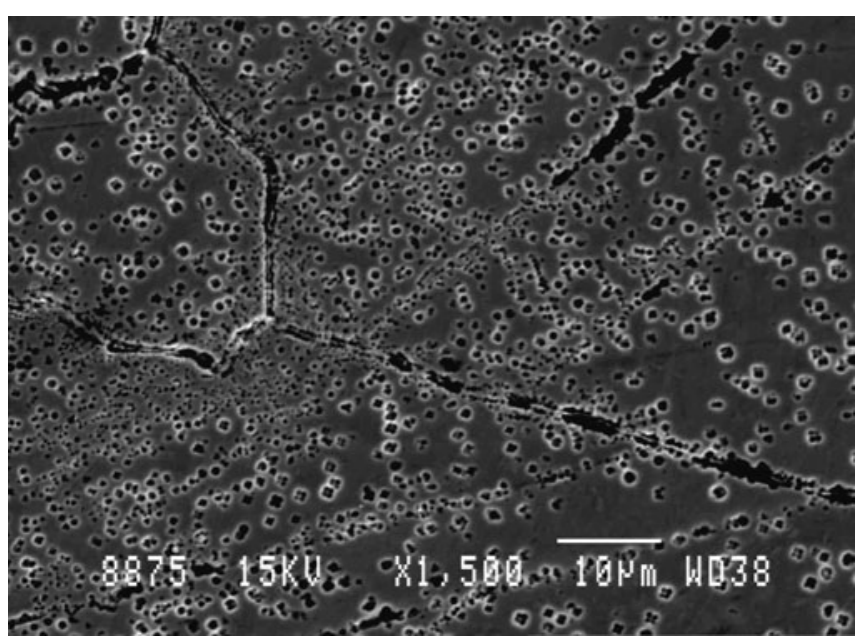

Figure 1. SEM microstructure of sample aged at $1073 \mathrm{~K}$ for $50 \mathrm{~h}$ showing extensive precipitation of coherent $\gamma^{\prime}$ intermetallic phase. it is appropriate to point out that minor changes in the modulus reflect in changes in the ultrasonic velocity and hence can be monitored.

From these results, it is evident that the ultrasonic velocity is more sensitive to the initial stages of the precipitation process. This is attributed to the fact that the ultrasonic velocities depend on the elastic moduli of the material, which are very sensitive to any compositional changes. There are several theories of precipitation hardening. According to the coherent lattice theory (Avener 1974) the alloy remains in a supersaturated condition after solution treatment and quenching, with the solute atoms distributed at random in the lattice structure. The process of precipitation of intermetallic phases upon ageing the solution annealed alloy can be divided into two stages: (i) incubation period, when the excess solute atoms tend to migrate to certain crystallographic planes, forming clusters or embryos of the precipitate; and (ii) precipitation, when these clusters form an intermediate crystal structure or transitional lattice, maintaining registry (coherency) with the lattice structure of the matrix. This phase will have lattice parameters different from those of the solvent, and as a result of the atom matching (coherency) there will be considerable distortion of the matrix. This distortion (strain-free) extends over a distance more than the size of a discrete (precipitate) particle. It is this distortion that interferes with the movement of dislocations and accounts for the strength during ageing. The first stage of the precipitation (i.e. incubation) involves only interatomic rearrangement which drastically influence the elastic moduli of the material and in turn the ultrasonic velocities. The depletion of the precipitate forming elements from the matrix leads to increase in the modulus of the matrix (Avener 1974). Hence, the response is faster for ultrasonic velocity and it also saturates during ageing of the solution annealed alloy. Further, as the coarsening of $\gamma^{\prime}$ phase and its conversion to acicular $\eta$ phase do not change the matrix composition, they do not influence the ultrasonic velocities. These observations are in line with those reported by Avener (1974) and Kumar et al (2006) for influence of precipitation of intermetallic phases on Young's modulus in other nickel base superalloys.

\section{Conclusions}

From the above study the following conclusions can be drawn:

(I) Ultrasonic can be used for monitoring very minor changes in elastic modulus.

(II) Elastic modulus is sensitive to minor chemical compositional changes occurring on heat treatment and can be monitored accurately by ultrasonic with very little or no sample preparation.

(III) Large increase in the precipitates leads to only minor changes in the elastic modulus. 


\section{Acknowledgements}

The authors would like to thank the Director, National Metallurgical Laboratory(CSIR), Jamshedpur, for his kind permission to carry out and publish this work. This work has been supported by AR\&DB (India) under sanction no. DARO/08/1061180/M/I.

\section{References}

Avener S H 1974 Introduction to physical metallurgy (Tokyo: McGraw Hill) 2nd ed., p. 192

Betteridge W and Heslop J (eds) 1974 The nimonic alloys (London: Edward Arnold) p. 178
Ho W F, Ju C P and Chen Lin J H 1999 Biomaterials 202115

Kumar A, Jayakumar T, Raj B and Ray K K 2003 Mater. Sci. Eng. A362 58

Kumar A, Jayakumar T and Raj B 2006 Philos. Mag. Letts 86579

Ledbetter H M 1983 Elastic properties in materials at low temperatures (ed.) R P Reed and A F Clark (Ohio: American Society for Metals (ASM)) pp 1-45

Long M and Rack H J 1997 Wear 205130

Majumdar P, Singh S B and Chakraborty M 2008 Mater. Sci. Eng. A489 419

McIntire P 1991 Non-destructive testing handbook: Ultrasonic testing (USA: American Society for Testing and Materials) 2nd ed., Vol 7

Moorthy R B, Jayakumar T and Rao K B S 2003 Int. Mater. Rev. 48 273

Zhao J C and Henry M F 2002 JOM 37 